\title{
“EL NIÑO" Y LA ESTRATEGIA NACIONAL DE CONSERVACIÓN
}

\section{"EL NIÑO" AND THE NATIONAL STRATEGY OF CONSERVATION}

\author{
Eduardo Franco*
}

La intervención de "El Niño" (EN) sobre la diversidad biológica en nuestro país, no se da siempre en forma directa. Una lluvia intensa, efecto climático de $\mathrm{EN}$ en determinada región, tendrá un efecto distimto sobre el suelo de una ladera, según ésta se encuentre provista de su cobertura vegetal o desprovista, por un proceso de deforestación, sobrepastoreo, explotación inadecuada, etc.

EN es un fenómeno cuya peligrosidad o efecto positivo sobre la diversidad biológica depende de las condiciones de vulnerabilidad o equilibrio de los sistemas y ambientes. Un ambiente degradado puede convertir una oferta ambiental - por ejemplo lluvias por efecto de EN - en una amenaza o peligro. Estos procesos de degradación y vulnerabilidad de determinados ambientes se encuentran relacionados con procesos socio-económicos de índole regional, nacional e intemacional. La presión que las poblaciones y los sistemas productivos ejercen sobre los recursos en determinadas regiones del país, se producen como consecuencia de una relación de subordinación entre distintos espacios geográficos y procesos económicos. Ello se manifiesta localmente, pero obedece a relaciones, estrategias y modelos de desarrollo que involucran al país en su conjunto.

En tal sentido, la Estrategia Nacional de

\footnotetext{
* Programa de Desastres

ITDG - Perú
}

Conservación de la Diversidad Biológica debe:

- Promover la investigación de factores que inciden en el hecho de que EN, siendo en muchos casos un factor al servicio de la diversidad biológica, se convierte en una amenaza de la misma.

- Promover proyectos y programas específicos y concretos de recuperación de medios degradados, en donde su condición convierte a EN en peligroso; de tal manera que, revirtiendo esos procesos, EN adquiera su papel positivo para la diversidad biológica. Estos proyectos y programas deben considerar los procesos sociales y económicos que se hallan en la base de los procesos de degradación ambiental y, en particular, la participación de las poblaciones que se beneficiarian directamente de ellos.

- La intervención de EN sobre la diversidad biologica no se da únicamente en los eventos extremadamente fuertes o extraordinarios (ejemplo 1982-83, 1997-98). Los efectos positivos o negativos se dan también en los eventos de menor intensidad (ejemplo 1972-73, 1986-87, 1991-93). EN es parte de la variabilidad climática interanual $e$ interdecadal. "Niños" leves que debieran ser únicamente favorables tienen también efectos desfavorables por la situación de los medios y sistemas. El tema de EN debe ser incorporado en la Estrategia Nacional de Conservación de la Diversidad Biológica. 
como un evento recurrente de distinta intensidad, regularmente presente en el territorio nacional, y no sólo en sus manifestaciones extraordinarias.

- La pérdida o conservación de la diversidad biológica, particularmente cuando se trata de la conservación in situ, está con frecuencia íntimamente relacionada al uso de tecnologías y la adecuación de las mismas al ambiente. La investigación, desarrollo y difusión de tecnologías adecuadas al ambiente y al patrimonio cultural de diversas regiones del país puede convertir la estrategia y práctica de la conservación en un proceso social ordinario, ligado a la producción y al desarrollo social, antes que aislado o extraño a las expectativas de las poblaciones humanas. La Estrategia Nacional de la Conservación de la Diversidad Biológica debe abordar de manera explícita este campo.

- El tema de los efectos de EN, según las condiciones en que se encuentren los ambientes y sistemas, es el mismo al que nos remite el tema de los desastres en el país y su relación con procesos de ocupación territorial, tipo de usos de los componentes de la diversidad biológica y de los recursos naturales en general, con los sistemas productivos y las políticas de desarrollo. La Estrategia Nacional de Conservación de la Diversidad Biológica debe incorporar, de manera manifiesta y explícita, el tema de los desastres en el país. La comunicación y vinculación entre temas, comunidades científicas y políticas, fortalecerá la capacidad de intervención del Estado y de la sociedad civil en el país sobre los procesos sociales económicos, políticos e institucionales, que inciden tanto en la conservación de la diversidad biológica, como en la reducción de los riesgos de desastres ecológicos y sociales propiamente dichos. Esta vinculación entre temas, comunidades científicas y diseño de políticas acrecentará la valía de la Estrategia Nacional de Conservación de la Diversidad Biológica ante la sociedad. El tema de EN es una oportunidad y espacio para que ello se produzca, y debe ser adecuadamente aprovechado. 